\title{
Flow between the train underbody and trackbed around the bogie area and its impact on ballast flight
}

\author{
J. Y. Zhu ${ }^{1,2^{*}}$, Z. W. $H u^{1}$ \\ ${ }^{1}$ Aerodynamics and Flight Mechanics Research Group, Faculty of Engineering and the Environment, \\ University of Southampton, Southampton SO17 1BJ, United Kingdom \\ 2 Institute of Railway and Urban Mass Transit, Tongji University, Shanghai 200092, China
}

\begin{abstract}
The aerodynamic behaviour of flow past a simplified high-speed train bogie including the ground underneath with ballast particles at scale 1:10 is studied numerically. It is found that the flow around the bogie is highly unsteady due to strong flow separations and flow interactions developed there. Generally, the ballast particles distributed inside the wheels are situated in the stronger turbulent flow and are subject to much higher aerodynamic forces than the particles located outside the wheels. Moreover, these aerodynamic forces increase when the ballast particles are located downstream of the bogie cavity and reach the peak values close to the bogie cavity trailing edge. Force time-series are produced based on the simulations of an array of the ballast particles in a wind-tunnel setup and it shows that the ballast flight is apt to happen as the rear part of the bogie cavity passing the ballast bed. When the ballast particles become airborne, the fluctuating forces generated increase significantly. Therefore, the stronger unsteady flow developed around the bogie cavity, especially in the cavity trailing edge region, will produce larger fluctuating forces on the ballast particles, which will be more likely to cause ballast flights for highspeed railways.
\end{abstract}

Keywords: ballast flight; flow behaviour; bogie area; aerodynamic forces

\section{Introduction}

High-speed railways are being developed rapidly around the world. Much progress has been made in the understanding of the aerodynamic phenomena associated with highspeed trains [1-5]. Recently, the flow behaviour and the corresponding aerodynamic noise generation mechanisms of a scaled isolated wheelset and simplified bogie have been investigated, which is found to be closely linked with the flow aerodynamics around the bogie [6,7]. Related to the underfloor carbody aerodynamics, a phenomenon of ballast flight under normal meteorological conditions occurs more frequently in associated with the operation of high-speed railways [8]. In addition to ballast flight, the physical

\footnotetext{
* Corresponding author.

E-mail addresses: zhujianyue@tongji.edu.cn (J. Y. Zhu), z.hu@soton.ac.uk (Z. W. Hu).
} 
background of ballast projection encountered on the railway networks has been carried out to investigate in Japan and Korea [9]. Ballast projection is revealed as a sporadic occurrence and causes impact damage to both train and track. Its developing starts from the aerodynamic initiation of motion of some larger ballast particles becoming airborne which later strike the vehicles, vehicle-mounted or trackside equipment and thereby bounce back into the trackbed and eject additional ballast particles. In France, ballast flight and projection have recently received more attention in view of the planned increase of train operational speed up to $350 \mathrm{~km} / \mathrm{h}$ [9]. The planned High Speed 2 (HS2) in the United Kingdom is to run at the speed around $360-400 \mathrm{~km} / \mathrm{h}$ in the ballasted track. Thus, improved understand of the flow behaviour of ballast flight would be useful for decision making and safety of the railway.

It is generally recognized that the damage generated on the rail running surface by wheelrail interaction is a major maintenance cost for any railway network [10]. For high-speed railways, the railhead damage known as 'ballast pitting' has become more serious [8]. This rail defect is caused by the small ballast particles becoming trapped between the rail running surface and the vehicle wheels. Thus, the sub-rail foundation experiences greater impact under heavy cyclic train loads particularly for high-speed railway lines. Due to such progressive deterioration, the residual deformation of the ballast is produced and leads to poor ride quality and loss of track support as a consequence of the voids formed between the sleepers and the ballast [11].

In order to understand the mechanism of the ballast flight, field experiments were carried out to investigate the aerodynamic and mechanical forces acting on ballast particles which were generated during the passage of a high-speed train [8]. Additionally, an analytical model was established to identify the factors causing the small ballast particles being ejected from the trackbed. It was found that ballast flight could arise from a combination of both aerodynamic and mechanical effects and the process was stochastic [8].

The aerodynamic loads on the trackbed causing ballast projection have also been investigated $[9,12]$. The wind tunnel experiments showed that the trackbed geometry, i.e. the type of sleepers and the level of the ballast bed surface, had a strong influence on the initiation and intensity of ballast particle dislodgement. However, obtained under 
idealized conditions in the laboratory, these results could not be correlated directly to realistic trackbed situations. Based on experiments with a ballast catapult dealing with the impact of particles on the ballasted trackbed, a linear relation between the number of ejected granules and the kinetic energy of the impacting grain has been found [9].

The basic characteristics of the flow between the underbody of a high-speed train and the ground have been studied numerically based on a turbulent Couette flow model to simplify the calculation [13]. The influence of the parameters (height of the gap, Reynolds number and roughness of the upper wall) on the equivalent surface roughness was analyzed. It was found that an equivalent roughness of the trackbed made of sleepers and ballast could be obtained based on this analytical method. However, configurations with more detail geometries between the train and the ground are needed for the flow calculation.

A full-scale computational fluid dynamics (CFD) simulation based on Reynolds-averaged Navier-Stokes (RANS) investigation of the ICE3 (inter-city express, German high-speed train) geometry using the nonlinear $k-\varepsilon$ turbulence model together with a wall function approach was performed. The results showed that thick boundary layers were developed considerably in the train underfloor region due to the generation of turbulence and secondary flow in the vicinity of the bogies. Additionally, the skin friction was increased significantly immediately downstream of the inter-car gap. The RANS results from this study didn't correspond well with the experimental measurements [9]. Improvement on CFD results is needed for better understanding of the train underbody aerodynamics and its influence on the ballast flight.

The mechanisms of the ballast flight or projection are not well understood $[8,9]$. Since the flow beneath a high-speed train running on a ballasted track is highly unsteady and turbulent, it is still very difficult to numerically predict the ballast flight or projection. The bogies and the train underbody cavities are the main components in the train underbody regions and contain many geometries exposed to flow with little or no streamlining, leading to complex flow structures which affect the flow behaviour around the trackbed. As a first step, the flow behaviour under the train carbody with some ballast particles attached on and close to the trackbed around the bogie region is investigated based on 
the numerical simulations performed for a simplified bogie situated in the bogie cavity with a ground underneath.

\section{Numerical Method}

Aerodynamically, high-speed trains are operating within the low Mach number flow regime, for example at $300 \mathrm{~km} / \mathrm{h}$ the Mach number is about 0.25 . The incoming flow simulated here is at low Mach numbers ( 0.09 corresponding to $30 \mathrm{~m} / \mathrm{s})$ and thereby the compressibility effects are small and therefore can be neglected to the hydrodynamic airflow field. Therefore, the unsteady incompressible Navier-Stokes equations are used to solve the flow field. The continuity and momentum equations in tensor notation are

$$
\begin{gathered}
\frac{\partial u_{i}}{\partial x_{i}}=0 \\
\frac{\partial u_{i}}{\partial t}+u_{j} \frac{\partial u_{i}}{\partial x_{j}}=f_{i}-\frac{1}{\rho} \frac{\partial p}{\partial x_{i}}+v \frac{\partial^{2} u_{i}}{\partial x_{j} \partial x_{j}},
\end{gathered}
$$

where $x_{i}$ represents the Cartesian coordinates in the three directions for $i=1,2,3 . p$ is the pressure, $\rho$ is the density, $v$ the kinematic viscosity, $f_{i}$ is the body force and $u_{i}$ the flow velocity components. Here $\rho$ and $v$ are constants for incompressible flow. The open source software OpenFOAM-2.2.1 is employed to solve the governing equations. A second-order accurate scheme is utilized for the convection and diffusion terms of the spatial derivatives and the temporal discretization follows a second-order fully implicit scheme. The pressure-velocity algorithm PIMPLE, combining PISO (pressure implicit with splitting of operator) and SIMPLE (semi-implicit method for pressure-linked equations) algorithms [14], is applied to solve iteratively the resulting discretized linearalgebra equation system. The delayed detached-eddy simulation (DDES) model based on the Spalart-Allmaras (S-A) turbulence model is employed for the current flow calculations. The S-A model is a one-equation model which solves a convection-diffusion equation for the modified turbulent kinematic viscosity, $\tilde{v}$. The transport equation is defined as

$$
\frac{\partial \tilde{v}}{\partial t}+u_{j} \frac{\partial \tilde{v}}{\partial x_{j}}=G_{v}-Y_{v}+\frac{1}{\sigma_{\tilde{v}}}\left[\frac{\partial}{\partial x_{j}}\left((v+\tilde{v}) \frac{\partial \tilde{v}}{\partial x_{j}}\right)+C_{b 2}\left(\frac{\partial \tilde{v}}{\partial x_{j}}\right)^{2}\right]
$$


where the three terms on the right-hand side are the production term, destruction term and diffusion term of the model variable $\tilde{v}$. Details of the formulations were introduced in [15].

As a hybrid technique, detached-eddy simulation (DES) combines unsteady Reynoldsaveraged Navier-Stokes (URANS) modelling in the near-wall regions to resolve the boundary layer, with large-eddy simulation (LES) in the massive separated outer flow regions to capture the large-scale structures [16]. The applications of DES in the fully turbulent mode have been confirmed by the benchmark problem results with different codes [17]. Delayed detached-eddy simulation has been developed to avoid grid-induced separation and preserve the RANS mode throughout the boundary layer. The CFD simulation results using DDES with layered grid show good agreement quantitatively with the experimental data [16].

\section{Simulation Setup}

The three-dimensional model at 1:10 scale consisting of a simplified bogie and a section of the train underbody and trackbed with ballast particles is displayed in Fig. 1 where the incoming flow with freestream velocity $U_{0}$ is also indicated. The simplified bogie is the same bogie as used in [7]. To reduce the simulation cost, the most representative components that generate vortex shedding and turbulent wake, such as the wheels, axles and the frame, are kept in the simplified bogie model. The axle has a diameter $(d)$ of 17.5 $\mathrm{mm}$ and the wheels have a diameter $(D)$ of $92 \mathrm{~mm}$. The wheelbase (centre-to-centre length of two axles) is $252 \mathrm{~mm}$ which is about 14 times the axle diameter. The carbody under-floor surfaces are $4 \mathrm{~mm}$ above the bogie horizontal central plane. Distributions of ballast particles (also at 1:10 scale) relative to the bogie and cavity are described in Fig. 2. There are two rows of ballast particles placed on the ground to represent the multiballast particles distributed at typical locations along the train running direction. The sixteen ballast particles located outside the wheels are named ' $1 \mathrm{~L}$ ' to '16L' starting from upstream of the bogie cavity; and accordingly, the other sixteen ballast particles situated inside the wheels are named from '1R' to '16R'. The distance of the gap between each ballast particle is illustrated in Fig. 2 and the particles of '4L' to '13L' and '4R' to '13R' are located underneath the bogie cavity region. The ballast particles have the same 
dimensions of $4 \mathrm{~mm}, 4 \mathrm{~mm}$ and $2 \mathrm{~mm}$ along the streamwise, spanwise and vertical directions, respectively. According to 1:10 scale, a gap of $25 \mathrm{~mm}$ between the wheelset and the ground is considered to represent the distance of the wheels above the ground when running on the rails. As an initial step, the rail and the sleepers are neglected.

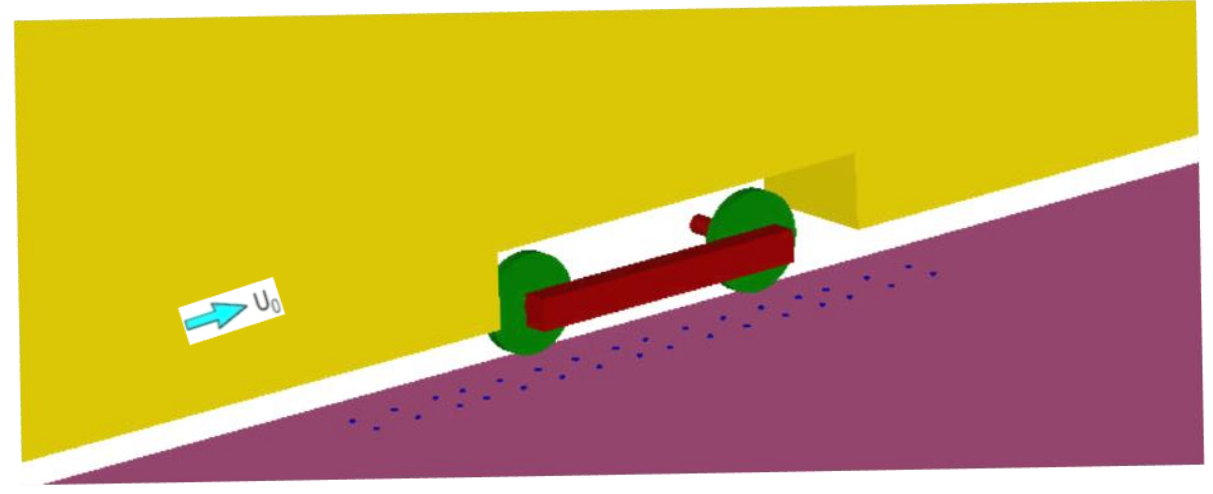

Fig. 1. Simplified model of train underbody and trackbed with ballast particles

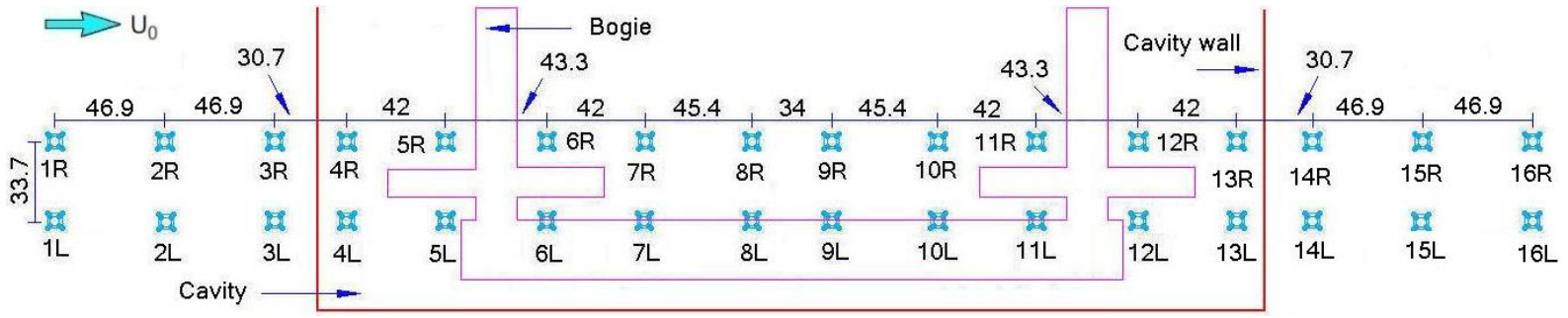

Fig. 2. Distributions of ballast particles around the bogie cavity area (1:10 scale and dimensions in millimetres, top view)

These bogie-inside-cavity cases are symmetrical about the axle mid-span plane where the influence of the three-dimensional flow from the wheel and frame is small; therefore it is reasonable to include only half of the geometry and make use of the symmetry to reduce the computational cost. As a wind-tunnel case, Fig. 3 illustrates the computational domain which has dimensions of $20.7 D, 11 D$ and $6.3 D$ (where $D$ is the wheel diameter) along the streamwise $(x)$, vertical $(y)$ and spanwise $(z)$ directions, respectively. Thus, the outlet and side boundaries are far enough away to have negligible influence on the flow developed around the bogie and the bogie cavity. 


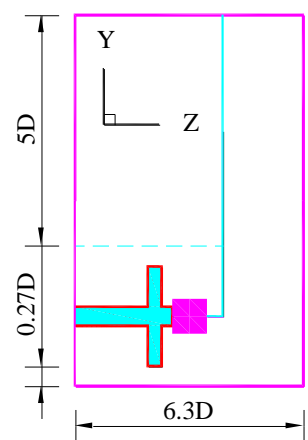

(a) Front view

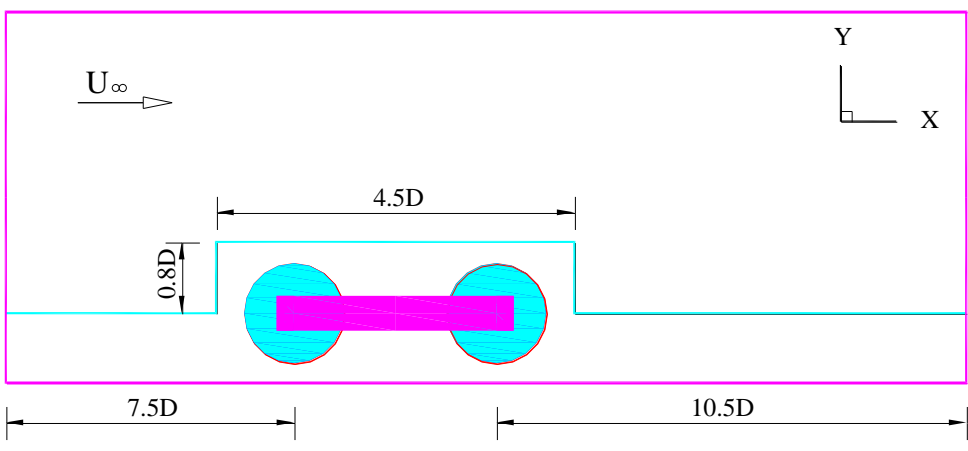

(b) Side view

Fig. 3. Sketch of the computational domain (not to scale)

A rigorous mesh refinement study for a complex geometry case is difficult to achieve because of the large calculations required for the unsteady flow. Based on the grid convergence study for the cylinder case [7], a fully-structured mesh is generated around all geometries with resolutions similar to the 'Baseline' grid of the cylinder case. The cell size on the axle surface is implemented as $0.42 \mathrm{~mm}$ around the perimeter and $0.88 \mathrm{~mm}$ in the spanwise direction. The maximum cell size on the wheel, frame and carbody surfaces is up to $1 \mathrm{~mm}$ and on the ballast particles is $0.5 \mathrm{~mm}$. As an example, the mesh generated around the bogie is displayed in Fig. 4. The distance from the bogie, carbody wall and ballast particle surfaces to the nearest grid point is set as $1 \times 10^{-5} \mathrm{~m}$ and stretched with a growth ratio of 1.1 inside the boundary layer. This yields a maximum value of $y^{+}$(the dimensionless first-cell spacing, $y^{+}=\frac{y u_{\tau}}{v}$ where $y$ is the distance from the wall, $u_{\tau}$ the friction velocity and $v$ kinetic viscosity) less than 1 for all cases which ensures that the boundary layer is resolved properly and the turbulence model employed can account for the low-Reynolds number effects inside the viscous sublayer. This grid generation strategy results in a fully block-structured mesh in the entire domain with a total number of grid points of 38.8 million for the current case with the ballast particles attached on the trackbed. 


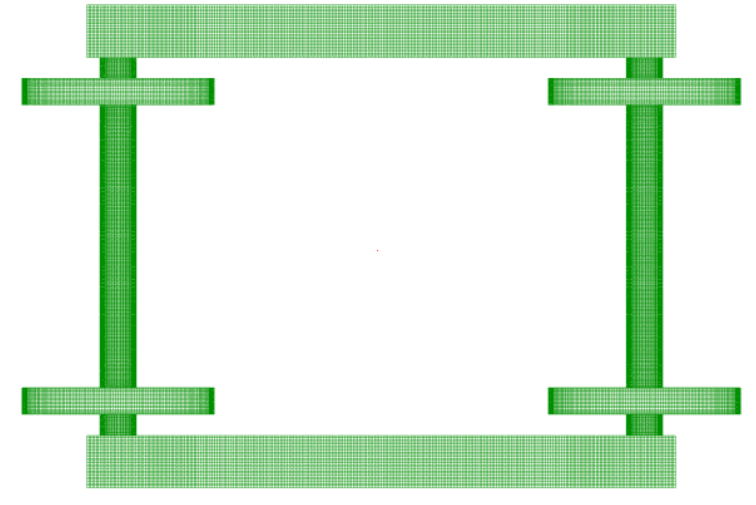

(a) Bogie surface

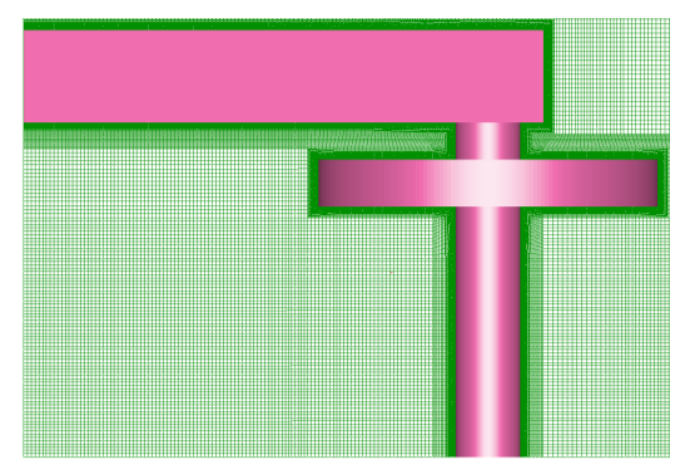

(b) Bogie horizontal mid-plane

Fig. 4. Structured mesh topology around the bogie ( $x-z$ plane)

The boundary conditions applied are as follows: the upstream inlet flow is represented as a steady uniform flow of $30 \mathrm{~m} / \mathrm{s}\left(U_{\infty}\right)$ with a low turbulence intensity. The top, bottom, axle mid-plane and side boundaries are specified as having symmetry boundary conditions which are equivalent to zero-shear slip walls; a pressure outlet with zero gauge pressure is imposed at the downstream outflow boundary; the wheelsets are assumed non-rotating and all solid surfaces including the carbody, bogie, ballast particles and ground are defined as stationary no-slip walls. Simulations are run with a physical timestep of $5 \times 10^{-6} \mathrm{~s}$ initially increasing to $1 \times 10^{-5} \mathrm{~s}$ which gives an adequate temporal resolution for the implicit time marching scheme used with a Courant-Friedrichs-Lewy (CFL) number of less than 1 within most part of the computational domain and a maximum value of 2 . The Reynolds number (based on the freestream properties and the axle diameter) of the current case is 36,000 , which is within the subcritical flow regime. The simulations were performed on the Iridis 4 clusters at the University of Southampton. For the case with the ballast particles attached on the trackbed, the calculations were parallelized over 352 processors and approximately $3.1 \times 10^{4}$ timesteps were required to reach a fully developed flow field. Thereafter, the collection of flow data were run further for around $6.8 \times 10^{4}$ timesteps to obtain a reasonable frequency resolution in data processing. Each timestep requires a physical time of $12 \mathrm{~s}$. The total time needed for the simulation was around 2 weeks continuous running.

The same meshing strategy, boundary conditions and numerical methods were employed for the isolated wheelset and bogie cases in which good agreements were achieved between numerical simulations and experimental measurements for the radiated far-field 
aerodynamic noise performed by the authors $[6,7,18]$. Thus, these simulation cases may improve the confidence of the current numerical calculations.

\section{Simulation Results}

In order to understand the flow behaviour around the simplified model consisting of a bogie, the bogie cavity and the trackbed with ballast particles, the properties of the DDES model, the calculation results of the instantaneous iso-surfaces of $Q$-criterion and the vorticity fields are analyzed to obtain an overview of the unsteady flow developed around the geometries; then, the fluctuating force coefficients of ballast particles are compared. Subsequently, when the ballast flight occurs, the flow characteristics of the airborne ballast particles are discussed.

\subsection{Properties of the DDES model}

In flow simulation using DES model, the mesh should be so designed as to ensure that the boundary layer region is modelled by RANS while LES is only switched on outside the boundary layer. In order to check the RANS/LES switching of the DDES scheme, Fig. 5 illustrates the radial profiles of the mean velocity, the model length scale ratio $\left(r_{d}\right)$, DDES function $\left(1-f_{d}\right)$ and the ratio of the modified length scale to wall distance $(\tilde{d} / d)$ at $\theta=75^{\circ}$ (measured anti-clockwise from the front stagnation point) at the locations of the axle mid-plane and wheel mid-span. In DDES model, the switch between RANS and LES is controlled by a redefined length scale $(\tilde{d})$ which depends not only on the cell wall distance and grid spacing but also on the time-dependent eddy-viscosity field. The delay function $f_{d}$ is given by

$$
f_{d} \equiv 1-\tanh \left(\left(8 r_{d}\right)^{3}\right),
$$

in which the model length scale ratio $r_{d}$ applied to any eddy-viscosity model becomes slightly more robust in the irrotational regions and is represented as

$$
r_{d} \equiv \frac{v_{t}+v}{\sqrt{U_{i, j} U_{i, j}} \kappa^{2} d^{2}},
$$

where $U_{i, j}=\partial U_{i} / \partial x_{j}$ is the velocity gradients and the molecular kinematic viscosity $v$ is employed to rectify the very-near-wall behaviour through keeping $r_{d}$ away from zero. The function $\left(1-f_{d}\right)$ approaches zero in the LES region. As shown in Fig. 5 , the axle mid- 
plane is defined at the half-length position of the axle segment inside the wheel, whereas the wheel mid-span represents the cutting surface from the mid-point along the axial direction. In terms of $r / D$ (the dimensionless distance to the wall surface) in the abscissa, the boundary layer (i.e. based on $U / U_{\infty}$ ) extends to 0.015 and the RANS/LES switching occurs (the location where $\tilde{d} / d$ becomes less than 1 ) around 0.04 at the upstream axle mid-plane. At the wheel mid-span, the boundary layer extends to 0.005 and RANS/LES switching occurs at 0.008 . It is shown that the RANS-LES interface remains well outside the boundary layer and the DDES delay function $f_{d}$ reaches 1 within the LES region. Thus, it can be confirmed that the RANS method is imposed over the entire boundary layer and the LES treatment is applied elsewhere when using the DDES model in the simulation.

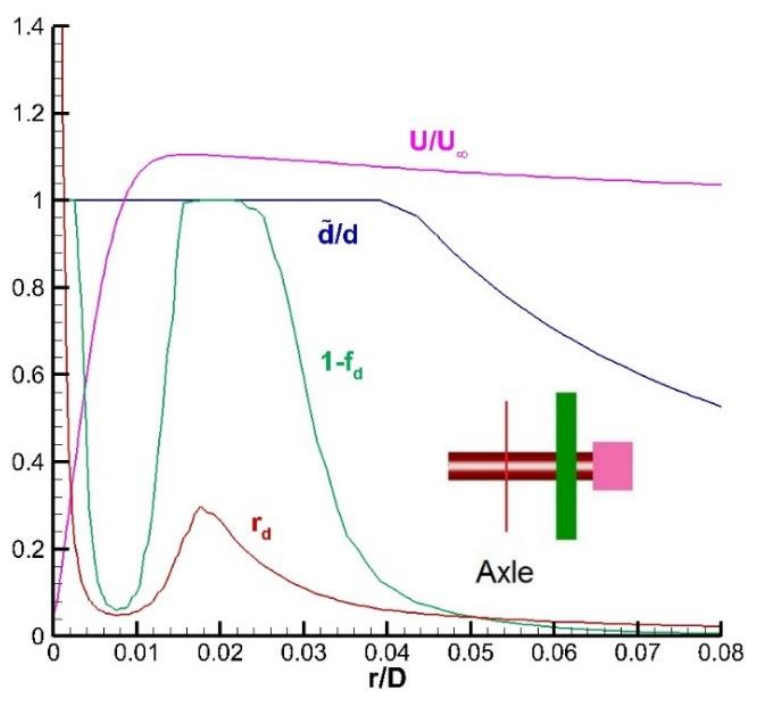

(a) Upstream axle mid-plane

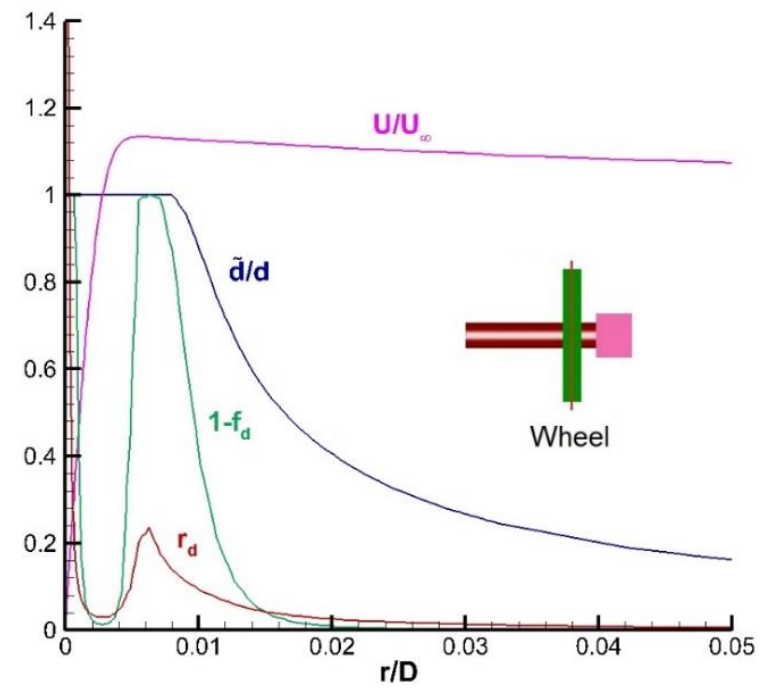

(b) Upstream wheel mid-span

Fig. 5. DDES model properties $\left(\theta=75^{\circ}\right)$

\subsection{Instantaneous flow field}

The vortical structures in the bogie wake represented by the iso-surfaces of the normalized $Q$-criterion at the value of 25 (based on $Q /\left[\left(U_{\infty} / D\right)^{2}\right]$, where $D$ is the wheel diameter) are visualized in Fig. 6 . They are coloured by the non-dimensional velocity magnitude. It can be seen that the various scales of vortices are formed between the upstream wheelset and cavity top wall as the various flow interactions and flow impingements occur there. Moreover, compared to the flow developed around the bogie, a higher level of flow-field unsteadiness is generated in the wake close to the cavity rear 
wall region where the vortices are convected downstream and impinge on the trackbed. Vortices developed on the trackbed within the boundary layer interact with the bogie cavity structures further downstream.
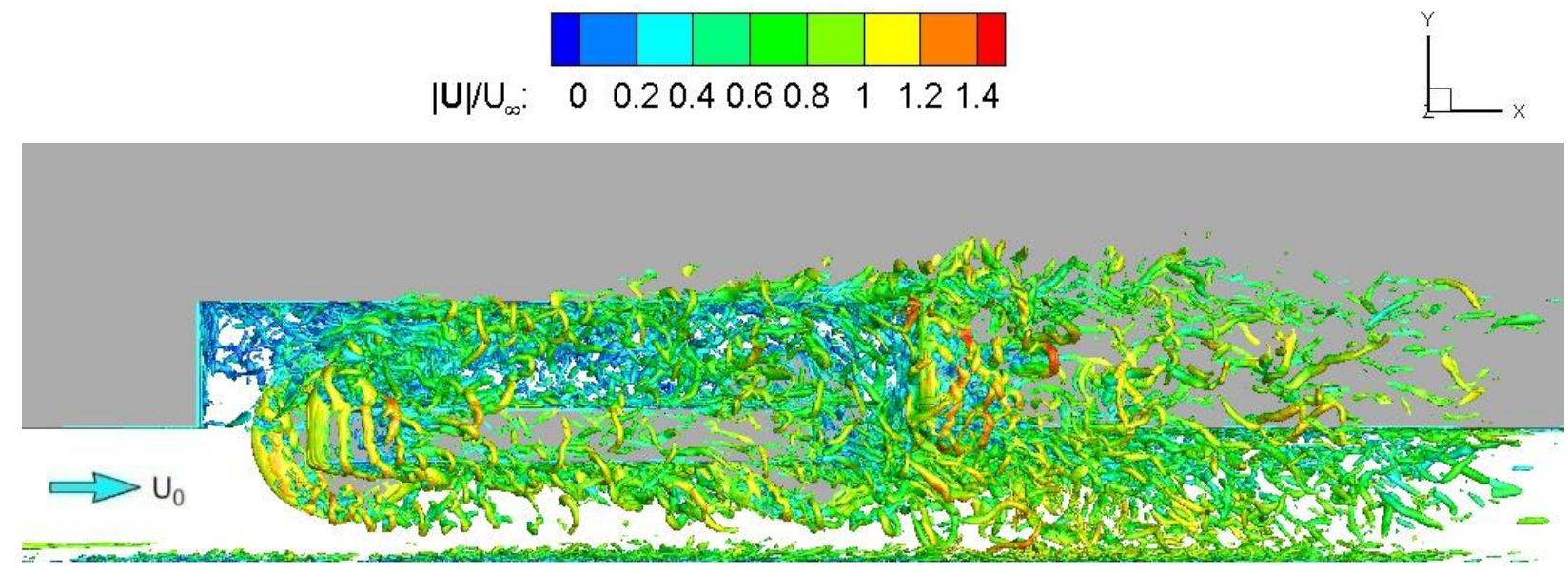

Fig. 6. Iso-surfaces of the instantaneous normalized Q-criterion

Fig. 7 shows the instantaneous non-dimensional spanwise vorticity field ( $\omega_{z}=$ $(\partial V / \partial x-\partial U / \partial y) D / U_{\infty}$, where $D$ is the wheel diameter) in the mid-span of the ballast particles located inside the wheels. It is shown that the shear layer shed from the cavity leading edge is bent upwards quickly in the streamwise direction. This shear layer travels downstream and interacts strongly with the flow separated from the upstream axle. Subsequently, all vortices are mixed up and impinge on the cavity top wall, leading to the unsteady flow with complex structures there. Additionally, it can be observed that the wake behind both the upstream and downstream axles is highly turbulent. As the downstream axle is sufficiently far from the upstream one, vortex shedding happens from both axles, leading to a flow pattern of "co-shedding". The downstream axle experiences the impingement of vortices shed from the upstream axle and the vortices developed behind the downstream axle are significantly deformed as they impinge on the cavity rear wall; thereby, all vortices are amalgamated behind the rear axle, leading to the chaotic behaviour of the downstream axle wake and the flow near the cavity rear corners highly irregular and unsteady. Some vortices generated around the downstream axle are convected downwards and impinge on the ground. Moreover, it can be seen that the vortices separated from the upstream ballast particles have a strong interaction with the flow developed around the ballast particles located behind them. 


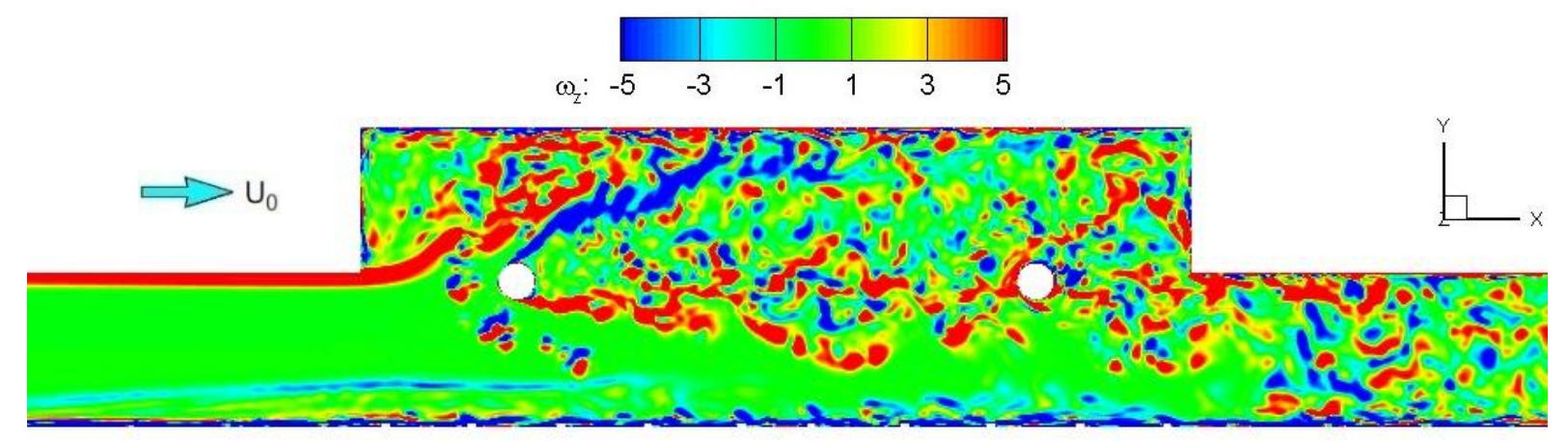

Fig. 7. Contours of instantaneous spanwise vorticity fields in vertical plane through the centre of ballast particles located inside the wheels (side view)

Contours of the instantaneous non-dimensional spanwise vorticity field $\left(\omega_{z}\right)$ in the midspan plane of the ballast particles located outside the wheels, which cut through the bogie frame, are displayed in Fig. 8. Similarly, a shear layer developed from the cavity leading edge is bent upwards and interacts with the vortices separated from the frame top surfaces; and thus, the vortical structure between the frame and the cavity top wall becomes highly turbulent due to flow impingement occurring there. The vortices developed and convected along the frame surface are separated at the frame ends, generating an unsteady wake region between the frame and the cavity wall. Again, some vortices are convected downwards from the frame bottom surface, interfering with the flow developed around the ground and the flow interactions are generated among the ballast particles.

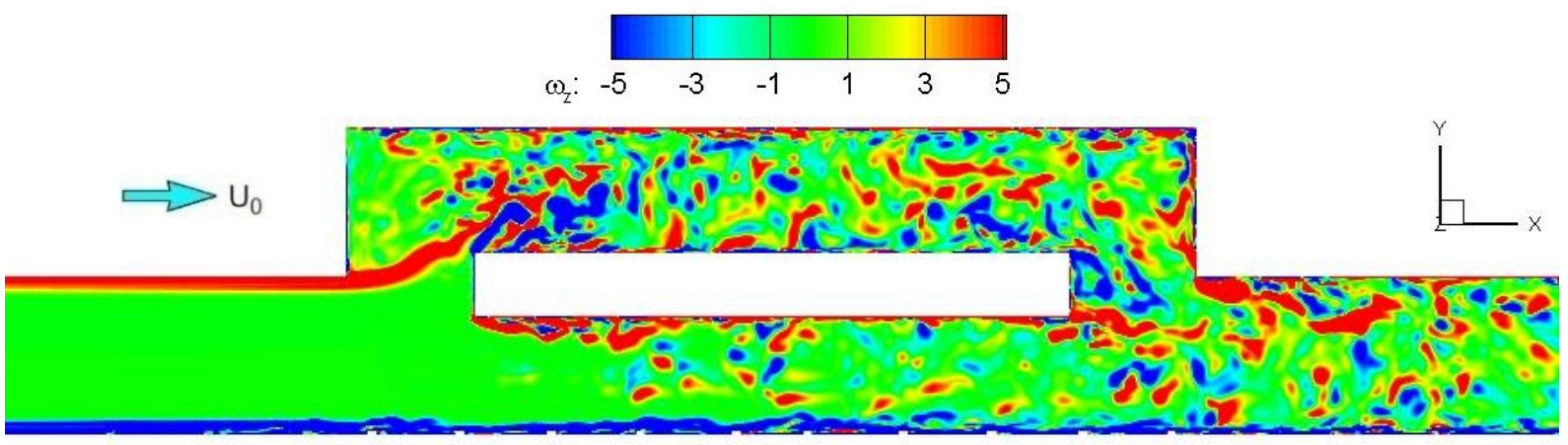

Fig. 8. Contours of instantaneous spanwise vorticity fields in a vertical plane through the centre of ballast particles located outside the wheels (side view)

Fig. 9 shows the instantaneous non-dimensional vertical vorticity field ( $\omega_{y}=$ $(\partial U / \partial z-\partial W / \partial x) L / U_{\infty}$, where $L$ is the ballast particle length of $\left.4 \mathrm{~mm}\right)$ at the ballast particle horizontal mid-plane from a bottom view. It can be seen that flow separation occurs at the side edge of the ballast particle. The vortices formed in the front ballast 
particles' wake are convected downstream and impinge on the rear ballast particles, causing more flow interactions around these regions and generating the unsteady flow along the trackbed.
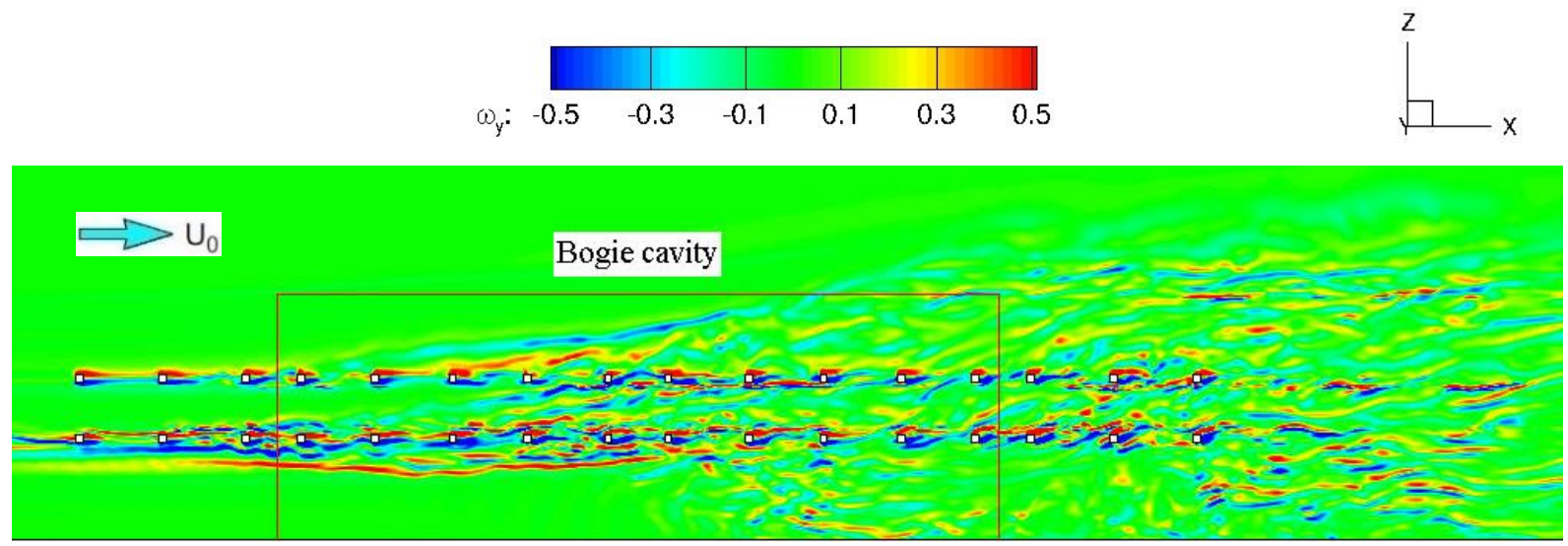

Fig. 9. Contours of instantaneous vertical vorticity fields through he ballast particle horizontal mid-plane (bottom view)

\subsection{Aerodynamic forces on ballast particles}

The power spectral densities (PSDs) of the fluctuating force coefficients of the ballast particles ('4L', '13L', '4R' and '13R') are presented in Fig. 10. Here the forces are nondimensionalised by $\left(\frac{1}{2} \rho_{0} U_{\infty}^{2} A\right)$ in which $\rho_{0}$ is the density in the undisturbed freestream and $A$ is the projected frontal cross-sectional area of the ballast particle $\left(A=8 e-6 \mathrm{~m}^{2}\right)$. As shown in Fig. 2, the particles of ' $4 \mathrm{~L}$ ' and ' $4 \mathrm{R}$ ' are distributed in front of the upstream wheelset; and the particles of '13L' and '13R' are located behind the downstream wheelset. The distance of the gap from the particles of ' $4 \mathrm{~L}$ ' (or ' $4 \mathrm{R}$ ') to the bogie cavity leading edge is identical to that between the particles of '13L' (or '13R') and the bogie cavity trailing edge. All these particles are situated underneath the bogie cavity region. Results show that various harmonic peaks are found in the lift, drag and side force spectra of the particle ' $4 \mathrm{~L}$ ', suggesting the flow developed around the particle located outside the wheels and close to the bogie cavity leading edge is dominated by the regular vortex shedding. Compared to the particle, the particle '4R' has a much larger amplitude in the spectra except at some harmonic peaks of '4L' as a consequence of the stronger turbulent flow development occurring inside the bogie region. Moreover, it shows that the particle '13R' has a higher level of the force spectra in the frequency range below $1 \mathrm{kHz}$ than the particle '4R'. This is because compared to the region of upstream wheelset near the cavity leading edge, a highly unsteady flow is generated around the area between the 
downstream wheelset and the cavity rear wall due to the strong flow impingement and flow recirculation developed there; and thereby the resulting turbulent flow induces the larger fluctuating forces on the ballast particles located in the cavity rear regions.

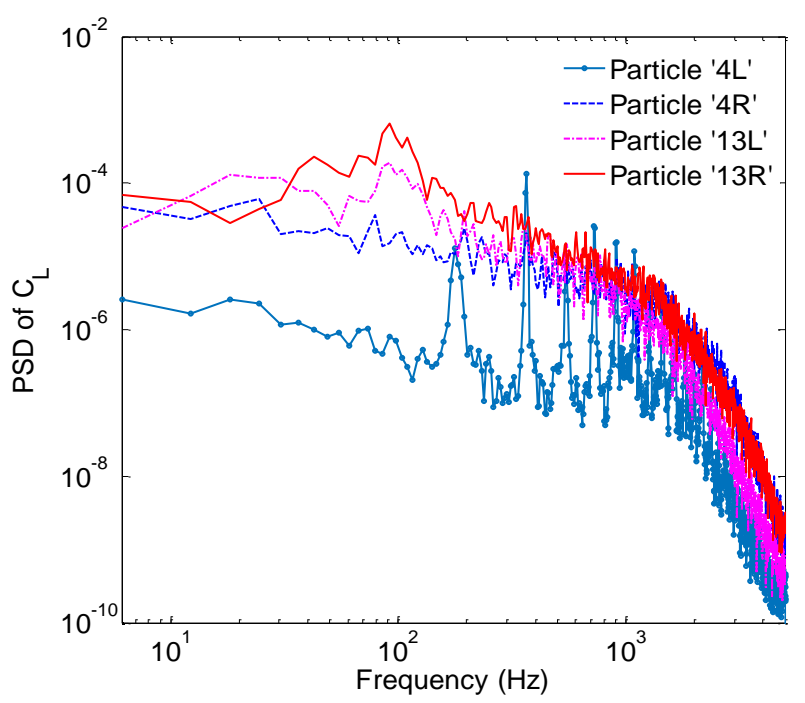

(a) Lift coefficient

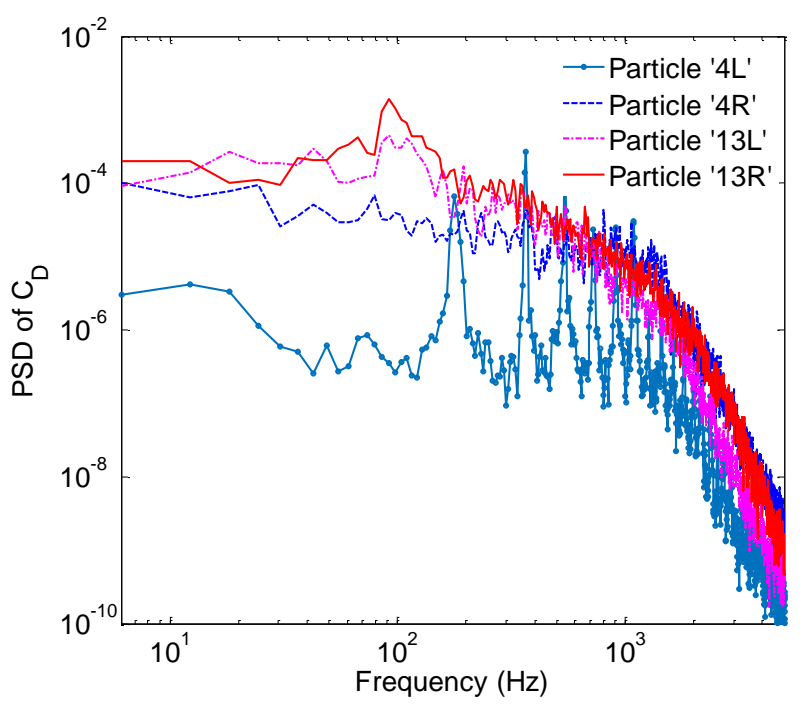

(b) Drag coefficient

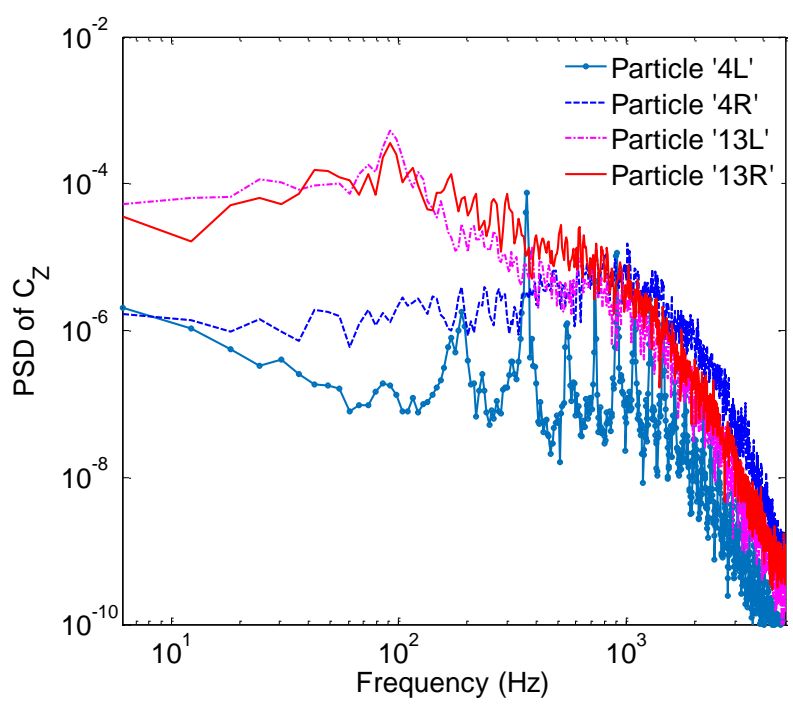

(c) Side force coefficient

Fig. 10. Power spectral densities of fluctuating force coefficients of the ballast particles

Comparisons of the root-mean-square fluctuating lift, drag and side force coefficients for all ballast particles are presented in Fig. 11. The values distributed on the consecutive ballast particles can also be treated as force time-series generated on one ballast particle assuming the train moving along a stationary ballast bed. Generally, the oscillations of the lift and drag coefficients on the ballast particles located inside the wheel are larger 
than those situated outside the wheel, owing to a much stronger unsteady flow developed inside the bogie region. Moreover, all forces increase for the ballast particles distributed downwards along the bogie cavity and reach the maximum values around the area of the bogie cavity trailing edge where the unsteady flow development is relatively stronger, as discussed earlier. Therefore, the fluctuating forces generated on the ballast particles are directly affected by the turbulent flow developed around them. The unsteady flow generated around the region between the rear wheelset and the bogie cavity rear wall will induce the large fluctuating forces on the ballast particles situated within it.

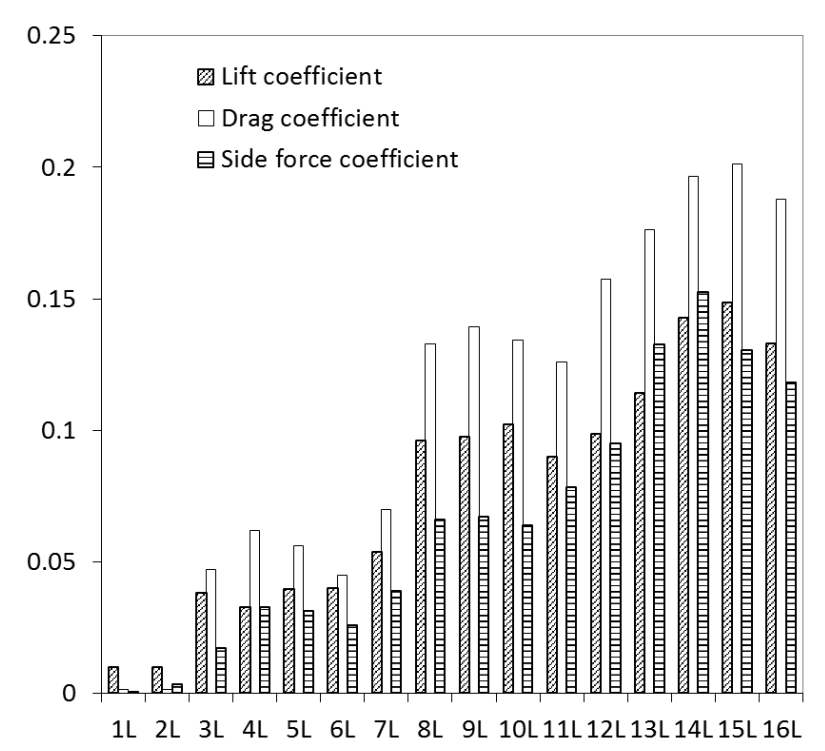

(a) Ballast particles outside the wheel

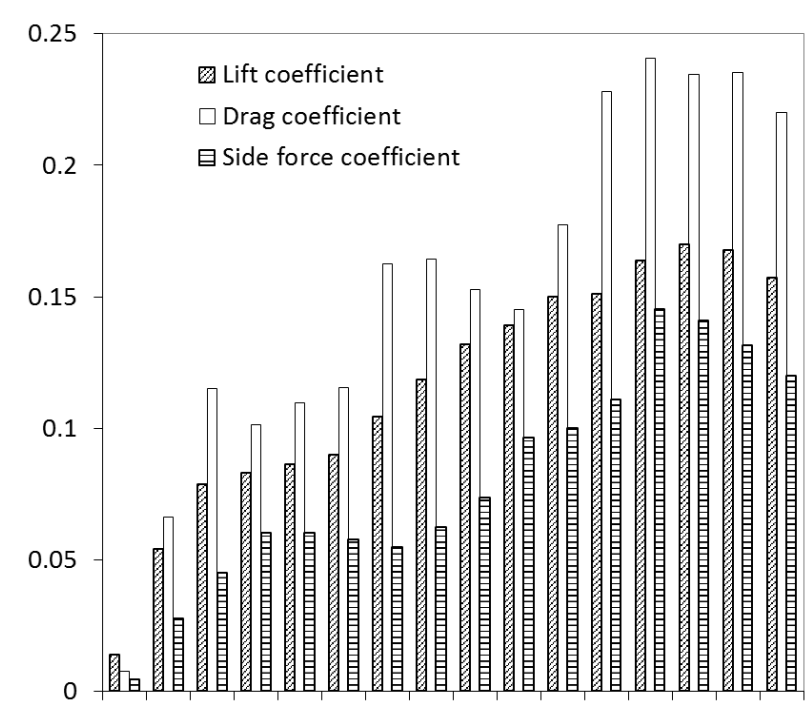

1R 2R 3R 4R 5R 6R 7R 8R 9R 10R11R12R13R14R15R16R

(b) Ballast particles inside the wheel

Fig. 11. Root-mean-square of fluctuating force coefficients of ballast particles

For the current numerical simulation of wind-tunnel case illustrated in Figs. 1-3, force signals of all ballast particles can be obtained individually which can start from the same time $t_{0}$ in the time series. Assuming the train moves along a stationary trackbed at a constant speed equal to the freestram velocity and passes by the first particle (' $1 \mathrm{~L}$ ' or ' $1 \mathrm{R}$ ') using time interval of $\Delta t_{1}$, the signals from $t_{0}$ to $\left(t_{0}+\Delta t_{1}\right)$ of the time series of first particle are picked. Then, the train passes by the second particle ('2L' or ' $2 \mathrm{R}$ ') using time interval of $\Delta t_{2}$, the signals from $\left(t_{0}+\Delta t_{1}\right)$ to $\left(t_{0}+\Delta t_{1}+\Delta t_{2}\right)$ of the time series of second particle are chosen and connected to the train-pass-by time series taken from the first particle, and so on. When the train passes by the nth particle using time interval of $\Delta t_{n}$, the corresponding signals of the time series of nth particle are kept and linked to the trainpass-by time series of the former adjacent particle. Thus, the signals of each ballast 
particle during the train passing by are added consecutively according to the pass-by intervals and a new time series signals are formed to simulate the whole bogie region passing by the ballast bed. Fig. 12 displays the time series of aerodynamic force coefficients of one ballast particle as train passing by the ballast particles located inside the wheels (the particles '1R' to '16R'). It is found that the fluctuating forces of the ballast particles are increased promptly when the front wheelset and, especially, the rear wheelset pass the ballast particles, which will make the ballast flight much more likely to happen .

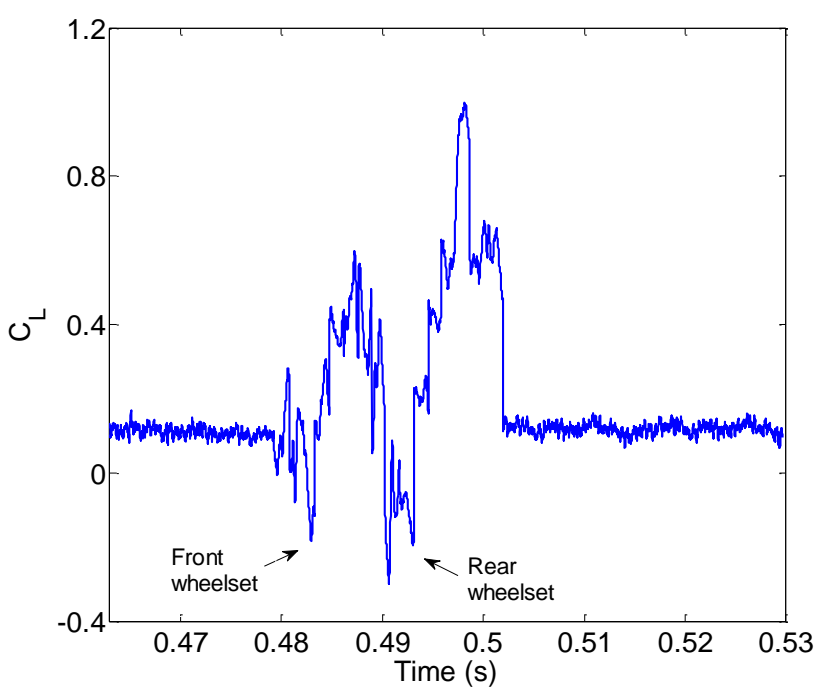

(a) Lift force coefficient

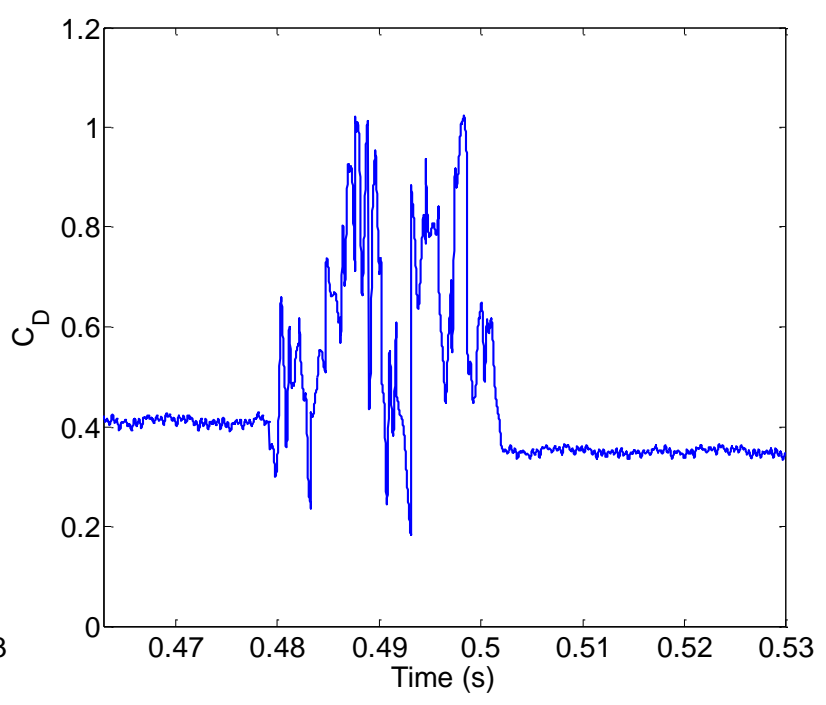

(b) Drag force coefficient

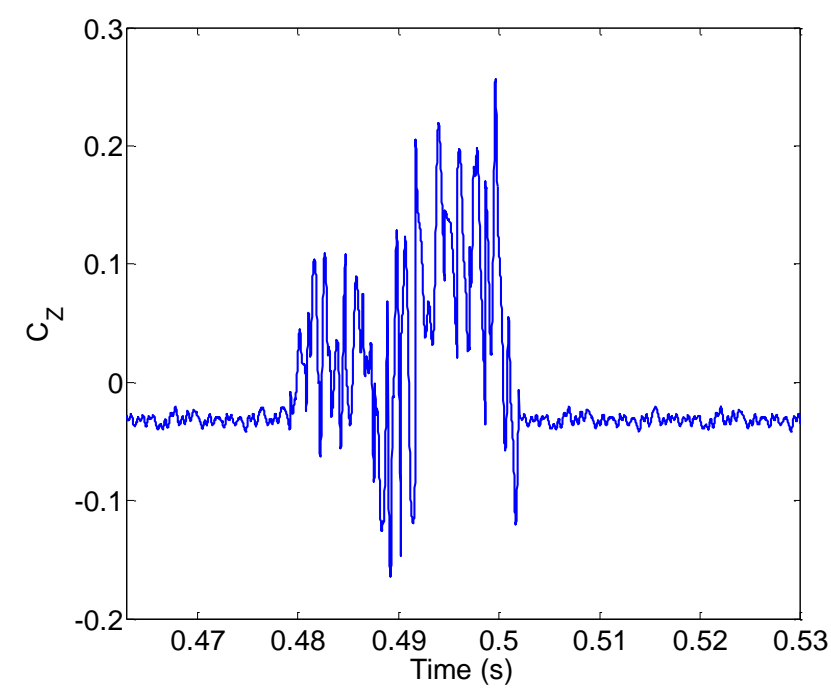

(a) Side force coefficient

Fig. 12. Time series of aerodynamic force coefficients of the ballast particles as the train passing by 


\subsection{Flow characteristics of the airborne ballast particles}

As discussed earlier, the wake of the upstream ballast particles will affect the flow field around the ballast particles located downstream. Since the ballast particles '13L' and '13R' are situated near the cavity rear wall where the turbulent flow is very strong as indicated by Figs. 6 and 7, the two particles '13L' and '13R' by removing the other ballast particles (displayed in Fig. 2) are used to investigate the flow bahaviour of the airborne ballast particles. Two cases with only the particles '13L' and '13R' attached to the ground and with a gap of $20 \mathrm{~mm}$ between the ballast particle centre and the ground are simulated. Fig. 13 displays that when the particle '13L' becomes airborne, the fluctuating force, especially the lift force (Fig. 13a), increases greatly. Table 1 also shows that the RMS value and the maximum value of the aerodynamic force coefficients of particle '13L' are much larger when it is airborne than it is attached to the ground. This is because the ballast particle leaves the trackbed and is situated in the energetic turbulent flow, and thus strong flow interactions are developed around all surfaces of the particle and generate the high force fluctuations on it. Compared to normal mass density of ballast particle, the fluctuating lift force is by several orders larger for the current ballast particle model, resulting in a large absolute aerodynamic force on the ballast particles. The aerodynamic behaviour of ballast particle '13R' located inside the wheels is similar to that of the particle '13L' outside the wheels when they become airborne in the present case.

\begin{tabular}{ccccccc}
\hline Coefficients & \multicolumn{2}{c}{ Drag } & \multicolumn{2}{c}{ Lift } & \multicolumn{2}{c}{ Side force } \\
\hline Cases & $\begin{array}{c}\text { Attached } \\
\text { to ground }\end{array}$ & $\begin{array}{c}\text { Gap of } \\
20 \mathrm{~mm}\end{array}$ & $\begin{array}{c}\text { Attached } \\
\text { to ground }\end{array}$ & $\begin{array}{c}\text { Gap of } \\
20 \mathrm{~mm}\end{array}$ & $\begin{array}{c}\text { Attached } \\
\text { to ground }\end{array}$ & $\begin{array}{c}\text { Gap of } \\
20 \mathrm{~mm}\end{array}$ \\
\hline RMS value & 0.183 & 0.296 & 0.133 & 0.498 & 0.136 & 0.191 \\
Mean value & 0.803 & 1.071 & 0.559 & -0.128 & 0.241 & 0.265 \\
Maximum value & 1.267 & 1.737 & 0.918 & 1.189 & 0.562 & 0.841 \\
\hline
\end{tabular}

Table 1. Root-mean-square, maximum and mean values of fluctuating force coefficients of ballast particle '13L' 


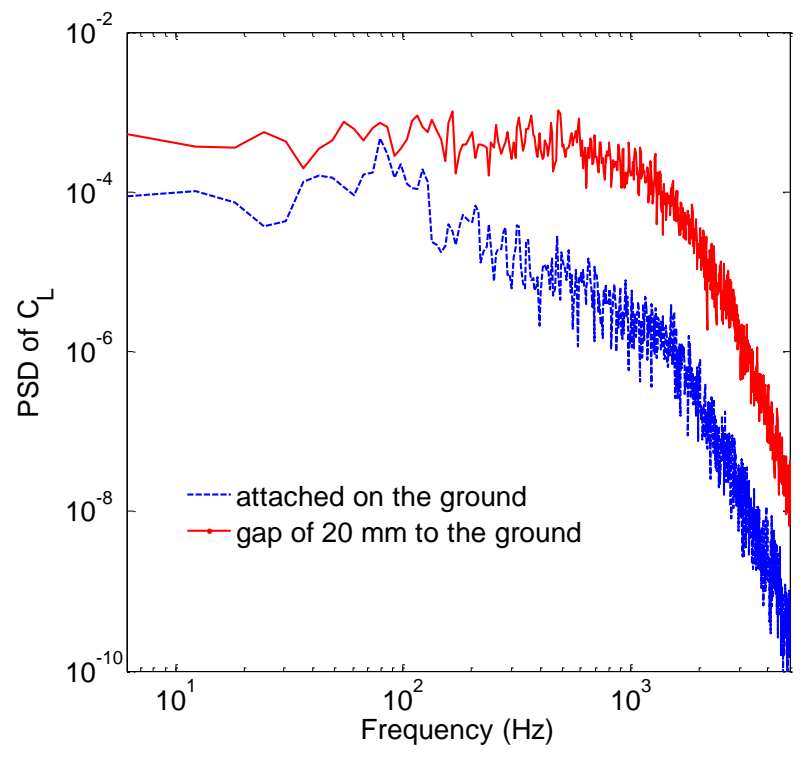

(a) Lift coefficient

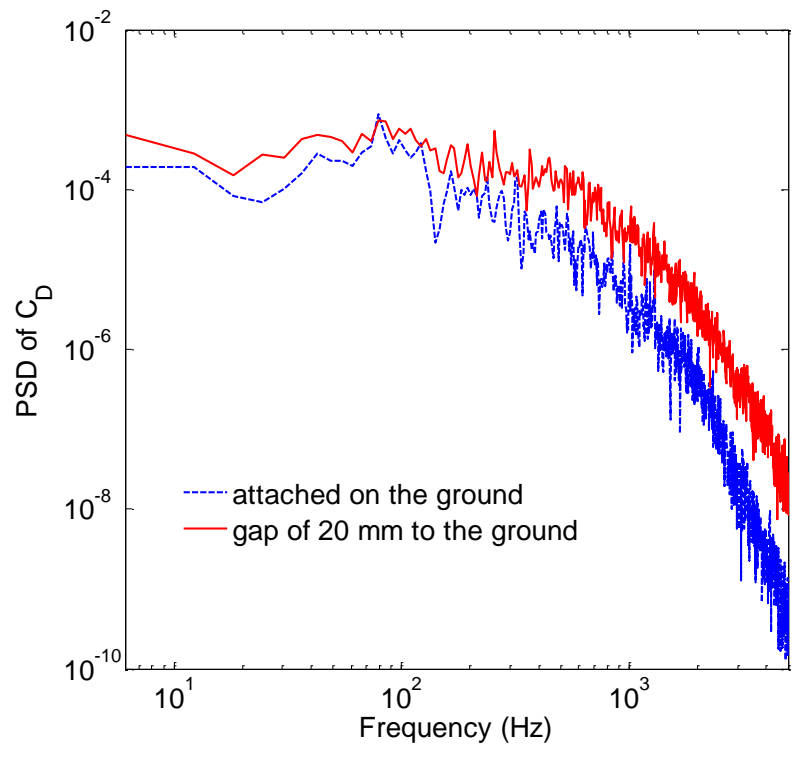

(b) Drag coefficient

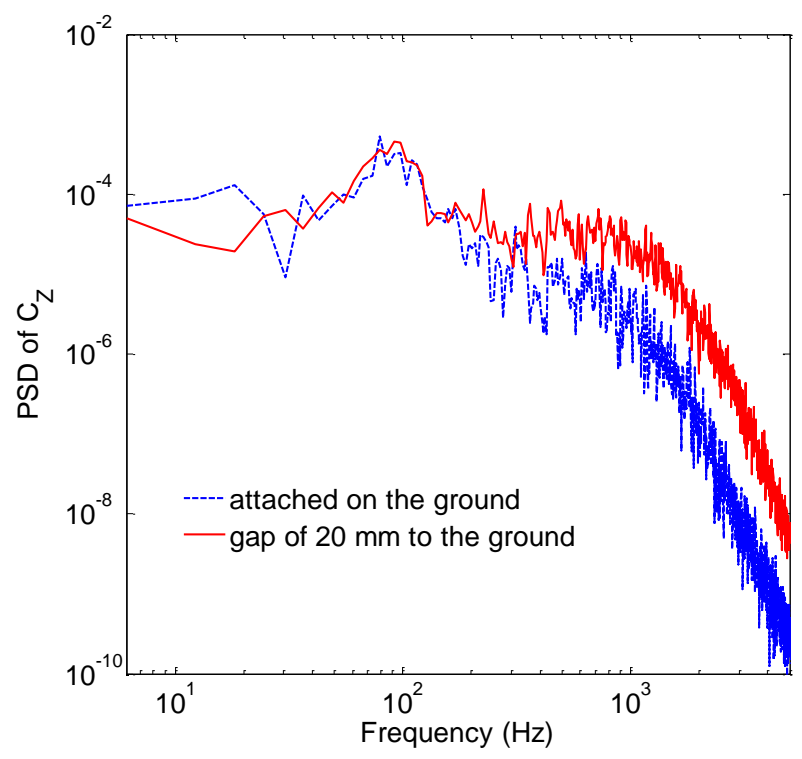

(c) Side force coefficient

Fig. 13. Power spectral densities of fluctuating force coefficients of ballast particle ' $13 \mathrm{~L}$ '

\section{Conclusions}

This study is a first step to understand the mechanism of the ballast flight by numerically predicting the interactions between the train underbody vortical flow and the ballast particles. The aerodynamic behaviour of the flow past the ballasted track beneath the train bogie has been investigated using DDES model. For the geometry of a bogie inside the cavity, a shear layer developed from the cavity leading edge has a strong interaction with the flow separated from the upstream bogie and cavity walls. All vortices are mixed 
up and convected downstream and impinge on the downstream geometries and cavity trailing edge regions. Thus, a highly irregular and unsteady flow is generated due to the strong flow impingements and flow interactions occurring there. Compared to the particles outside the wheels, the particles inside the wheels have much larger amplitudes in the fluctuating force spectra since more highly unsteady flow is developed inside the bogie area. As stronger flow interactions are generated around the region between the downstream wheelset and the cavity rear wall, larger fluctuating forces are induced on the ballast particles there than those situated around upstream wheelset near the cavity leading edge in most of the frequency range. Therefore, the ballast flight is more likely to occur when the rear wheelset and the bogie cavity trailing edge pass by the ballast particles. When the ballast particles become airborne, the fluctuating forces increase greatly since the particles are situated in the unsteady flow with more flow interactions which produces larger fluctuating forces on them. The findings based on the current numerical cases are helpful to understand the aerodynamic behaviour of ballast flight for an actual bogie at full scale passing by the ballasted trackbed.

Note that for high-speed train in reality, the Reynolds number is much higher. The presence of turbulent inflow and more detailed geometries will lead to more complex flow structures which will affect the ballast flight. Moreover, the highly turbulent flow developed around the trackbed will result in a stochastic behaviour on the ballast flight. The pressure pulse generated from the approaching train coupled with the severe mechanical vibration of the trackbed may play a key role in the initiation of the ballast flight. These factors need to be accounted for in future work.

\section{Acknowledgements}

This work was supported by the Engineering and Physical Science Research Council (EP/K03765X/1). Data supporting this study are openly available from the University of Southampton repository at http://doi.org/10.5258/SOTON/D0033. This work benifitted from discussions with Prof. William Powrie whose contributions are greatly appreciated. The numerical simulations were performed on the Iridis4 cluster managed by the University of Southampton. The first author is grateful for the support from Shanghai Key Lab of Vehicle Aerodynamics and Vehicle Thermal Management Systems. 


\section{References}

[1] Baker C.J., The flow around high speed trains. Journal of Wind Engineering and Industrial Aerodynamics, 2010, 98: 277-298.

[2] Baker C.J., A review of train aerodynamics Part 1-Fundamentals. The Aeronautical Journal, 2014, 118: 201-228.

[3] Hemida H., Krajnovic S., LES study of the influence of the nose shape and yaw angles on flow structures around trains. Journal of Wind Engineering and Industrial Aerodynamics, 2010, 98: 34-46.

[4] Jönsson M., Haff J., Richard H., Loose S., Orellano A., PIV investigation of the flow field underneath a generic high-speed train configuration. Euromech Colloquium 509, Vehicle Aerodynamics, External Aerodynamics of Railway Vehicles, Trucks, Buses and Cars, Germany, 2009.

[5] Schetz J.A., Aerodynamics of high speed trains. Annual Review of Fluid Mechanics, 2001, 33: 371-414.

[6] Zhu J.Y., Hu Z.W., Thompson D.J., Flow simulation and aerodynamic noise prediction for a high-speed train wheelset. International Journal of Aeroacoustics, 2014, 13(7\&8): 533-552.

[7] Zhu J.Y., Hu Z.W., Thompson D.J., Flow behaviour and aeroacoustic characteristics of a simplified high-speed train bogie. Proceedings of the Institution of Mechanical Engineers Part F: Journal of Rail and Rapid Transit, 2016, 230(7): 1642-1658.

[8] Quinn A.D., Hayward M., Baker C.J., Schmid F., Priest J.A., Powrie W., A full-scale experimental and modelling study of ballast flight under high-speed trains. Proceedings of the Institution of Mechanical Engineers Part F, Journal of Rail and Rapid Transit, 2009, 224: 61-74.

[9] Kaltenbach H.-J., Gautier P.-E., Agirre G., Orellano A., Schroeder-Bodenstein K., Testa M., Tielkes T., Assessment of the aerodynamic loads on the trackbed causing ballast projection: results from the DEUFRAKO project aerodynamics in open air (AOA). Proceedings of the World Congress on Rail research, South Korea, 2008.

[10] Cannon D.F., Edel K.-O., Grassie S.L., Sawley K., Rail defects: an overview. Fatigue \& Fracture of Engineering Materials \& Structures, 2003, 26(10): 865-886. 
[11] Zhu J.Y., Thompson D.J., Jones C.J.C., On the effect of unsupported sleepers on the dynamic behaviour of a railway track. Vehicle System Dynamics, 2011, 49: 1389-1408.

[12] Deeg P., Jönsson M., Kaltenbach H.-J., Schober M., Weise M., Cross-comparison of measurement techniques for the determination of train-induced aerodynamic loads on the trackbed. BBAA VI International Colloquium on: Bluff Bodies Aerodynamics \& Applications, Italy, 2008.

[13] García J., Crespo A., Berasarte A., Goikoetxea J., Study of the flow between the train underbody and the ballast track. Journal of Wind Engineering and Industrial Aerodynamics, 2011, 99: 1089-1098.

[14] OpenFOAM, http://www.openfoam.com (accessed on March, 2015).

[15] Spalart P.R., Allmaras S.R., A one-equation turbulence model for aerodynamic flows. AIAA Paper 92-0439, 1992.

[16] Spalart P.R., Deck S., Shur M.L., Squires K.D., Strelets M.Kh., Travin A.K., A new version of detached-eddy simulation, resistant to ambiguous grid densities. Theoretical and Computational Fluid Dynamics, 2006, 20:181-195.

[17] Spalart P.R., Shur M.L., Strelets M.Kh., Travin A.K., Initial noise predictions for rudimentary landing gear. Journal of Sound and Vibration, 2011, 330: 4180-4195.

[18] Zhu J.Y., Aerodynamic noise of high-speed train bogies. PhD Thesis. University of Southampton, UK, 2015. 